Research Paper

\title{
Clinico-Pathological Correlation of $\beta$-Catenin and Telomere Dysfunction in Head and Neck Squamous Cell Carcinoma Patients
}

\author{
Swatishree Padhi ${ }^{*}$, Arka Saha ${ }^{*}$, Madhabananda Kar ${ }^{2,5}$, Chinmoy Ghosh ${ }^{1}$, Amit Adhya ${ }^{3}$, Manas Baisakh ${ }^{4}$, \\ Nachiketa Mohapatra ${ }^{4}$, Shriram Venkatesan ${ }^{6}$, Manoor Prakash Hande ${ }^{6}$ and Birendranath Banerjee ${ }^{\bowtie}$ \\ 1. Molecular Stress and Stem Cell Biology Group, School of Biotechnology, KIIT University, Bhubaneswar, Odisha-751024, India \\ 2. Department of Surgical Oncology, Kalinga Institute of Medical sciences, Bhubaneswar, Odisha-751024, India. \\ 3. Department of Pathology, Kalinga Institute of Medical Sciences, KIIT University, Bhubaneswar, odisha-751024, India \\ 4. Department of Pathology, Apollo Hospitals, Bhubaneswar, Odisha-751004, India. \\ 5. Department of Surgical Oncology, Apollo Hospitals, Bhubaneswar, Odisha-751004, India \\ 6. Genome Stability Laboratory, Yong Loo Lin School of Medicine, Department of Physiology, National University of Singapore, Singapore 117597 \\ ${ }^{*}$ Authors contributed equally
}

Corresponding author: Birendranath Banerjee, Assistant Professor, KIIT School of Biotechnology, KIIT University, Bhubaneshwar-751024, Odisha, India: E-mail: bnbanerjee@kiitbiotech.ac.in, phone: +91- 9090840042. Fax: 0674-2378776

(c) Ivyspring International Publisher. This is an open-access article distributed under the terms of the Creative Commons License (http://creativecommons.org/ licenses/by-nc-nd/3.0/). Reproduction is permitted for personal, noncommercial use, provided that the article is in whole, unmodified, and properly cited.

Received: 2014.05.02; Accepted: 2014.07.04; Published: 2015.01.15

\begin{abstract}
Background: Tumorigenesis is a complex process of accumulated alteration in function of multiple genes and pathways. Wht signalling pathway is involved in various differentiation events during embryonic development and is conserved in various species.

Objective: A multicentre collaborative initiative is undertaken to study the occurrence, prognosis and molecular mechanism of HNSCC (Head and Neck Squamous Cell Carcinoma) which is highly prevalent in eastern parts of India. From a large cohort of HNSCC tissue repository, 67 cases were selected for multi-parametric investigation.

Results: 67 cases showed stable $\beta$-catenin expression. We have seen correlation, if any, of the transcription factor - $\beta$-catenin, telomere maintenance and shelterin complex proteins - TRF2, Rapl and hTert with respect to tumor differentiation and telomere dysfunction. Immunohistochemistry of $\beta$-catenin protein showed stable and high expression in tumor when compared to stroma. MDSCC (Moderately Differentiated Squamous cell carcinoma) cases expressed nuclear expression of $\beta$-catenin in invasive fronts and showed increased genomic instability. Higher frequency of Anaphase bridges was observed ranging from $<3 \%$ in normal cut margin to $13 \%$ in WDSCC (Well differentiated squamous cell carcinoma) and 18\% in MDSCC (Moderately differentiated Squamous cell carcinoma). There was significant decrease in telomere length in MDSCC $(<4)$ when compared to the normal cut margin samples $(<7)$. Quantitative Real Time-PCR confirmed a significant correlationship between stable $\beta$-catenin expression and poor clinical and pathological outcome.

Conclusion: The Stabilisation and accumulation of $\beta$-catenin was significant and correlated well with de-differentiation process as well as prognosis and therapy outcome of the patients in the cohort. Expression status of molecular markers such as $\beta$-catenin, hTert, TRF2 and RAP1 correlate significantly with the process of tumorigenesis and prognosis and may play a role in therapeutic management of Head and neck patients.
\end{abstract}

Key words: HNSCC, Genomic instability, Wnt/ $\beta$-catenin, Dedifferentiation, MDSCC, WDSCC. 


\section{Introduction}

\section{Head and neck squamous cell carcinoma (HNSCC) occurrence and recurrence}

With more than 500,000 new cases diagnosed each year, Head and Neck Squamous Cell Carcinoma (HNSCC) ranks among the 10 most common cancers worldwide. India, compared to the developed countries, has a higher rate of incidents due to the habits of tobacco chewing and smoking. ${ }^{[1-4]}$ The reason for limited survival of the HNSCC patients is the higher number of patients presented at an advanced disease stage, lack of suitable marker for early detection and failure to efficiently respond to the chemotherapy and minimal residual disease (MRD).[2, 5, 7] Thus to target HNSCC efficiently, improvement on conventional therapy is urgently required.[1]

Tumorigenesis is a complex process of accumulated alteration of multiple genes and pathways. More than $80 \%$ of adenomas and carcinomas have mutations in various growth promoting developmental genes resulting in acquisition of a growth advantage phenomenon for maintenance and metastasis. ${ }^{[6,7]}$ One of these is the canonical Wnt cascade. Notably, in many of the same tissues where the Wnt cascade controls stem cells, cancer ensues upon dysregulated activation of this pathway. $[8,9,43]$

\section{Various factors leading to tumorigenesis}

\section{The Wnt / $\beta$-catenin signalling pathway}

The Wnt signalling pathway is involved in various differentiation events during embryonic development and is conserved in various species. It leads to tumor formation when activated aberrantly. ${ }^{[8,9]}$ In the absence of signalling molecule (Wnt), free cytoplasmic $\beta$-catenin gets phosphorylated in the axin (destruction) complex leading to ubiquitination and degradation in the cytoplasm. ${ }^{[8,10]}$ As Wnt binds to the cell surface receptors, $\beta$-catenin escapes the degradation complex of axin, stabilizes and accumulates in the cytoplasm and translocates to the nucleus, where it binds with the transcription factors. viz., $\mathrm{T}$ cell factor (TCF)/lymphoid enhancer factor (Lef), leading to the upregulation of the wnt targeted genes. ${ }^{[11,12]}$ Evidence till date reveals that the genes that are helpful in occurrence and development of cancer are the targets of the Wnt/ $\beta$-catenin pathway and are usually upregulated as a consequence of the deregulation of the signalling molecules. ${ }^{[13]}$ Thus wnt $/ \beta$-catenin play a crucial role in determining the process of carcinogenesis.

\section{Genomic instability}

Telomere (protective end of chromosomes) determines genome integrity.[16, 17] Specific group of hexaproteins called shelterin (TRF1, TRF2, Rap1,
TIN2, POT1, and TPP1) with additional specific non-telomeric protein plays a vital role in cellular DNA damage response by protecting, regulating the telomere and maintaining the integrity of the genome.[14, 15] Telomere dysfunction leads to perturbation in various cell signalling pathways including the Wnt $/ \beta$-catenin pathway. ${ }^{[13,19]}$ Telomere attrition signals the DNA damaging sensors triggering the onset of genomic instability. [18] A common indicator and cause of chromosome instability is the formation of anaphase-bridge, where chromosomes are pulled towards both the poles of the centriole during segregation. ${ }^{[19]}$ These bridges then break due to mitotic stress and again form in the next interphase, giving rise to breakage-fusion-bridge cycles. ${ }^{[20]}$ DNA damage induces double stranded breaks (DSBs) which are difficult to repair, however they typically fuse back together to restore chromosomal structure. This misligation of two non-matching ends may result in the formation of Anaphase- Bridge and chromosomal instability. ${ }^{[25]}$ The consequential genomic instability is associated with aggressiveness and poor prognosis of the disease. ${ }^{[24]}$ In carcinoma, increased aggression is related to increase in the gradation of dysplasia resulting in poor prognosis. So it becomes important to study the underlying mechanism of telomere dysfunction with the aggressiveness of tumors.[25]

\section{Telomerase and its role in tumorigenesis}

Telomerase is a known regulator of telomere maintenance and several cofactors positively and negatively regulates the transcription of telomerase gene.[26, 16] Previously it has been reported, that hTert is an indirect transcriptional target of $\beta$-catenin of the Wnt developmental pathway. ${ }^{[26,28]}$ hTert, being the key player and specific to cancer cells, is also regulated by Wnt/ $\beta$-catenin in those cells. ${ }^{[13]}$ Recently it has been shown that hTert serves as a cofactor for the $\beta$-catenin transcriptional complex and hence plays a significant role in Wnt/ $\beta$-catenin pathway. ${ }^{[29]}$ Mutations in $\beta$-catenin results in upregulation of hTert expression facilitating stabilization of the telomeres thus providing a significant hall mark for tumorigenesis.

The dynamics of $\beta$-catenin and telomere regulation is not completely clear. In order to study the correlation if any in between these two pathways we designed a multi parametric molecular pathological approach to relate observational findings of the protein expression and molecular changes.

\section{Materials and method}

In the present study, 67 HNSCC cases were selected from a library of large tissue repository with clinical information and analysed for stable $\beta$-catenin expression and then categorised according to their 
histopathological grading. Only those tissues which had stable $\beta$-catenin expression and complete clinical and prognostic information were selected. The differentiation grades were characterised as lower grade $(n=4)$, well differentiated $(n=29)$ and moderately differentiated $(n=34)$ squamous cell carcinoma. Grade specific analysis was done for stable $\beta$-catenin in stromal and tumor areas in the histopathological sections. Three important telomere maintenance proteins (TRF2, Rap1 and hTert) were also studied by immunohistochemistry and correlated with $\beta$-catenin. Patients with poor prognosis $(n=10)$ and higher correlationship of $\beta$-catenin and shelterin proteins were further investigated for genome instability by telomere length analysis (Quantitative fluorescent insitu hybridisation) and Anaphase bridges. Q-PCR analysis was done for the randomly selected 12 cases and repeated three times to check the correlationship between the $\beta$-catenin expression level and the clinical outcome of the patient.

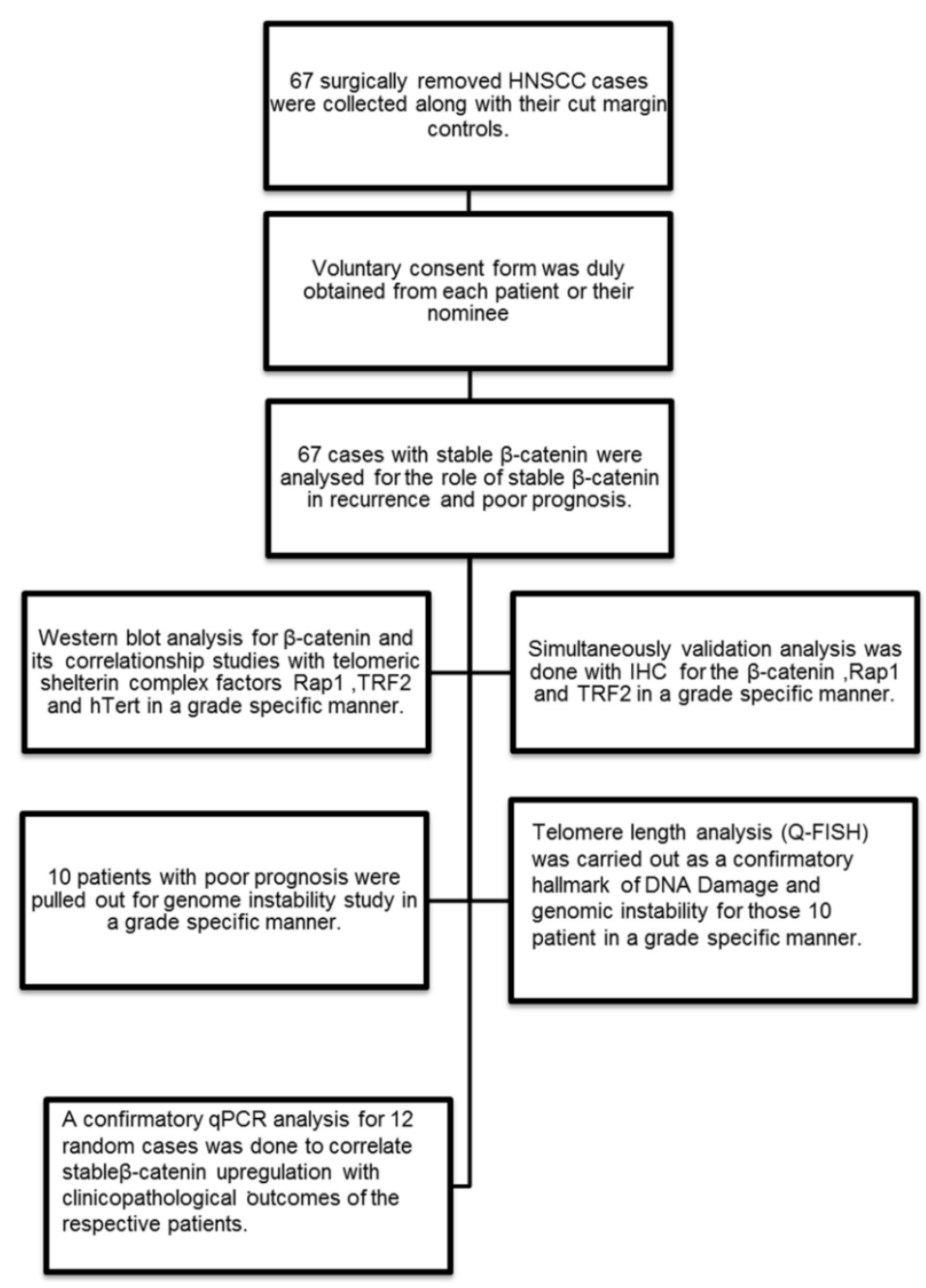

Figure A: Flow chart showing the experimental methodology and study design.

\section{Patient sample collection}

Human tissue samples from patients with Head and Neck squamous cell carcinoma were acquired at the time of surgical removal of the tumor tissue across the cut margin area. Cut margin tissues were collected as normal counterparts of an individual case. Tissues were collected in Protease Inhibitor Cocktail solution and $4 \%$ Formaldehyde for whole tissue lysate preparation for Western blot analysis and tissue processing for the immunohistochemistry respectively. The samples were kept at $15-25^{\circ} \mathrm{C}$ overnight and then stored at $-80^{\circ} \mathrm{C}$ storage. Voluntary consent form was duly signed from the patient or their nominees. The human sample collection was followed strictly as per institutional ethical board guidelines. The study was approved by institutional ethics committee.

\section{Protein extraction /SDS-PAGE/western blot}

Lysates were prepared from the tumor and their corresponding normal tissue by using the conventional homogenization method using Liquid nitrogen by RIPA Lysis buffer $(50 \mathrm{mM}$ Tris-Cl $\mathrm{pH}$ 7.4,150Mm Nacl,1\% NP-40, $0.25 \%$ sodium desoxycholate,1mM EDTA,1mM PMSF, $1 \mathrm{mM}$ $\mathrm{Na}_{3} \mathrm{VO}_{3}, 1 \mathrm{mM}$ EGTA,1x PIC,MQ). $50 \mu \mathrm{g}$ of protein lysates were separated using $12 \%$ SDS-Polyacrylamide Gel Electrophoresis. The gel was then subjected to electro blotting on PVDF membrane. The membrane was blocked at 5\% skimmed milk in PBST for $1 \mathrm{hr}$ at RT and then probed with the primary antibody (1:2000) for $1 \mathrm{hr}$ at RT or at $4^{\circ} \mathrm{C}$ overnight. The membrane was washed with PBST and probed with secondary antibody (1:4000) for $2 \mathrm{hrs}$ at RT. The blots were visualized by enhanced chemiluminescence using X-ray film (Kodak, India). [33, 34]

\section{Immunohistochemistry}

Immunohistochemistry analysis was done for HNSCC tissue sections taken over poly-L-lysine coated slides. The tissue sections were taken from formalin fixed, paraffin embedded blocks. ${ }^{[28,33]}$ Immunostaining was done by the use of Biogenex Super Sensitive TM Polymer-HRP IHC Detection system (REF: QD400-60KE). Antigen retrieval was done by the use of microwave method in $10 \mathrm{mM}$ Sodium citrate buffer $\mathrm{pH} 6.0$ for 20 minutes. The sections were then blocked with 3\% BSA in TBST for $2 \mathrm{hrs}$ at RT and then probed with primary antibody (1:2000) for $1 \mathrm{hr}$ at RT or at $4^{\circ} \mathrm{C}$ overnight. Peroxide block was performed for 15 minutes in dark at RT to avoid the non-specific signals. Sections 
were washed and then incubated with the secondary antibody (1:4000) conjugated with HRP for 90 minutes at RT followed by washing and treatment with DAB chromogen for 5 minutes at RT. Sections were washed and counterstained with haematoxylin. ${ }^{[3,34]}$ Imaging of the stained slides was done using Leica (DM 2000) bright-field microscopy.

\section{Anaphase bridge analysis}

Analysis of the frequency of anaphase bridges is commonly considered as an indicator of chromosomal instability. ${ }^{[35]}$ The tissue sections were taken from formalin fixed, paraffin embedded blocks. ${ }^{[35,34]}$ The sections were then stained with Haematoxylin and Eosin. Anaphase bridges were counted per thousand dividing cells both for tumor and the cut margin sections.

\section{Telomere length analysis on tissue sections by peptide nucleic acid- fluorescence in situ hy- bridization}

Paraffin embedded tissue sections of $3-4 \mu \mathrm{m}$ thickness were deparaffinised in 100\% Xylene followed by dehydration in $100 \%$ ethanol, and subsequent incubation in $1 \mathrm{M}$ sodium thiocyanate at $80^{\circ} \mathrm{C}$ for $8 \mathrm{~min}$. Slides were then incubated in 1\% pepsin. ${ }^{[35]}$ The tissue sections were then hybridized with $\mathrm{Cy} 3$ conjugated PNA telomere probe. Stringent washes were performed and the slides were counterstained with DAPI. Images were captured with Zeiss Axioplan 2 imaging microscope (Carl Zeiss, Gottingen, Germany) and the telomere signals were quantitated using the ISIS software (Metasystems, Altusseheim, Germany). The fluorescent intensity was calculated as arbitrary fluorescent intensity the software.

\section{RNA extraction and quantitative real time PCR}

Total RNA was extracted from the frozen stored tissues with TRIsure reagent (BIOLINE) in accordance with the manufacturer's instructions reverse transcription was performed in total volume of $20 \mu \mathrm{l}$ using $2 \mu \mathrm{g}$ of total RNA by TETRO cDNA synthesis kit (BIOLINE). Real time PCR was carried out on the CFX Connect $^{\mathrm{TM}}$ (BIORAD) for $\beta$-catenin using KAPA SYBR ${ }^{\circledR}$ FAST qPCR Kit Master Mix (2x) Universal (KapaBiosysytems). $\beta$-actin was used as the housekeeping gene. $\beta$-catenin PCR primers are designed based on human $\beta$-catenin mRNA sequence. The primer sequences are as follows: Forward 5'-GCTTTCAGTTGAGCTGACCA-3' and reverse 5' CAAGTCCAAGATCAGCAGTCTC- $3^{\prime}$. As an internal control, a fragment of human $\beta$-actin was amplified using the following primers: Forward 5'-TCAC CCACACTGTGCCCATCTACGA-3' and reverse
5'-CAGCGGAACCGCTCATTGCCAATGG-3'. PCR conditions were set as per manufacturer's instruction. Quantification was performed using the $2^{\Delta \Delta C T}$ method. The absolute levels of $\beta$-catenin mRNA were normalized to that of $\beta$-actin mRNA. The HNSCC tissues as well as their respective cut margins were compared with the lower grade leukoplakia case to check the status of differentially expressed $\beta$-catenin gene in each case.

\section{Statistical analysis}

\section{Quantification of western results}

The results obtained from western blotting were quantified using the VisionWorksLSTM software and the net upregulation and downregulation of each of the 5 antibodies for 67 (33 all 5, additional 34 $\beta$-catenin) cases were recorded.

Also grade specific percentage upregulation and downregulation for each of the 5 antibodies were recorded and a comparative graph was plotted using GraphPad Prism ${ }^{\mathrm{TM}}$.

\section{Quantitative immunohistochemistry}

Quantification of chromogen intensity in the immunohistochemical sections stained with the following antibodies, $\beta$-catenin, Rap1 and Trf2, was performed by ImmunoRatio ${ }^{\mathrm{TM}}$ and ImmunoMembrane $^{\mathrm{TM}}$ software. ${ }^{[36]}$

\section{Pathological analysis}

The histopathological Analysis for the considered cases was verified by two independent pathologists in a double blinded method. Any kind of discrepancy in analysis was resolved by a third independent pathologist. All the margins that were considered were not free of tumor. Infiltrations were verified by two independent pathologists. More than 1000 tumor cells were analysed per sample to decrease the experimental error.

\section{Results}

\section{Figure 1: Western blot and protein expression studies}

Expression levels of the proteins of interest, viz., $\beta$-catenin, TRF2, hTERT and Rap1, were studied for 67 tumor samples which showed stable $\beta$-catenin. The western data showed that not only the tumor samples but also the cut margin showed stable expression of $\beta$-catenin Figure 1A. Data obtained from quantitative analysis of western results were plotted and it was found to confirm the above observation of net stabilization for all the proteins under consideration in the tumor as well as their cut margin areas shown in Figure 1B. 


\section{Immunohistochemistry studies}

IHC of $\beta$-catenin showed section specific marked stabilization of the signals Figure 1C. Non-infiltrated regions showed no signal of tumorigenic $\beta$-catenin Figure 1C (I). Regions having infiltrated tumor cells had membranous. $\beta$-catenin expression Figure 1C (II) Regions of the tumor having moderately differentiated/poorly differentiated cells showing nuclear localization of the $\beta$-catenin as can be seen in the inset. Figure 1C (III).

\section{Figure 2: Comparative analysis of different grades of tumor}

A comparative analysis of the proteins of interest in WDSCC (Well differentiated squamous cell carcinoma) and MDSCC (Moderately Differentiated Squamous cell carcinoma) was performed by Immunohistochemistry. It was found differential ex-

A)

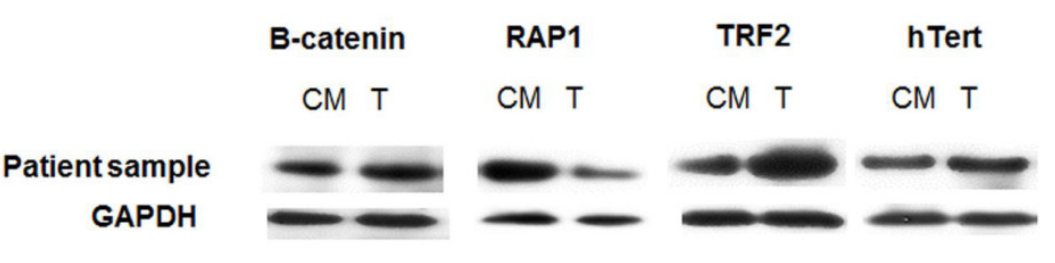

B)

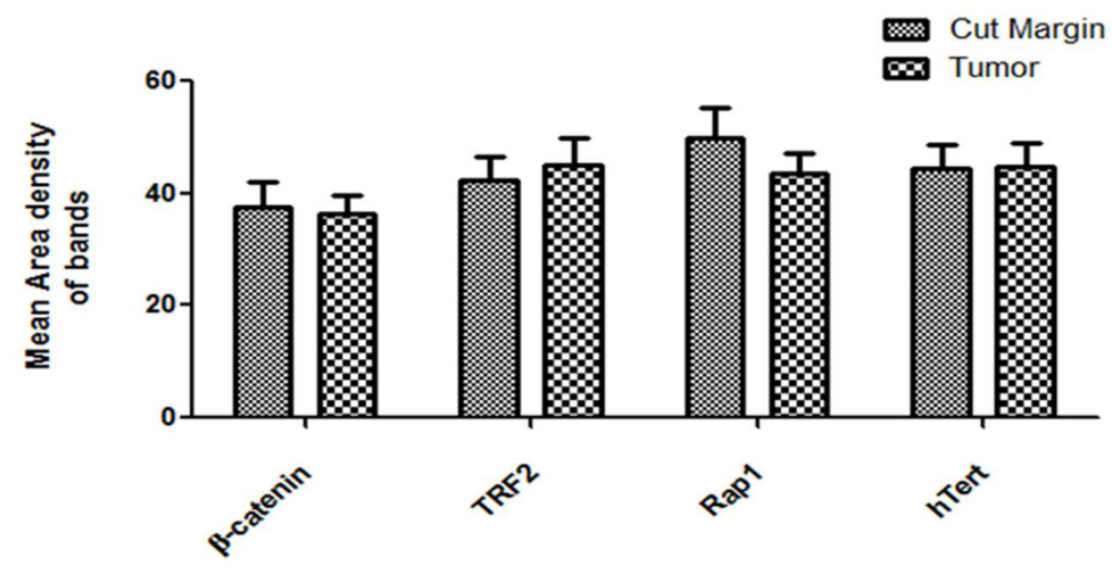

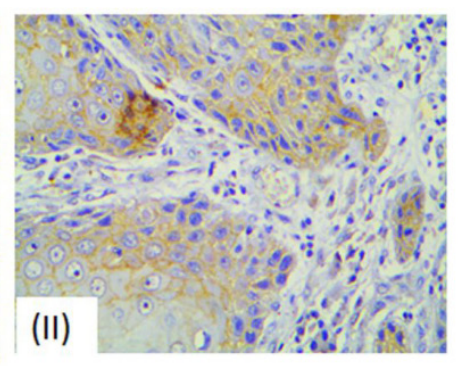

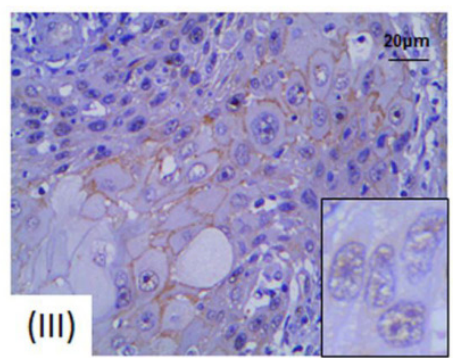

Figure 1: The expression level of proteins $\beta$-catenin, hTert, Rapl and TRF2 probed by respective antibodies in western blotting. (A) Western blot analysis shows expression level of the $\beta$-catenin, TRF2, Rapl and hTert in human samples in compared with GAPDH as loading controls. (B) Graphical representation of increased protein levels of $\beta$-catenin, hTert, Rapl and TRF2 in 33 patient sample cohort which was found to be stable in tumor tissue as well as their cut margin irrespective of their differentiation criteria of categorization analysed by VisionWorksLSTM. Error bars represent SE.(C) Immunohistochemistry validation of $\beta$-catenin showed section specific marked stabilization of the signals. (I) Non-infiltrated regions showed no signal of tumorigenic $\beta$-catenin.(II) Regions showing infiltrated tumor cells having membranous $\beta$-catenin expression.(III) Regions tumor showing nuclear localization of the $\beta$-catenin as can be seen in the inset. 


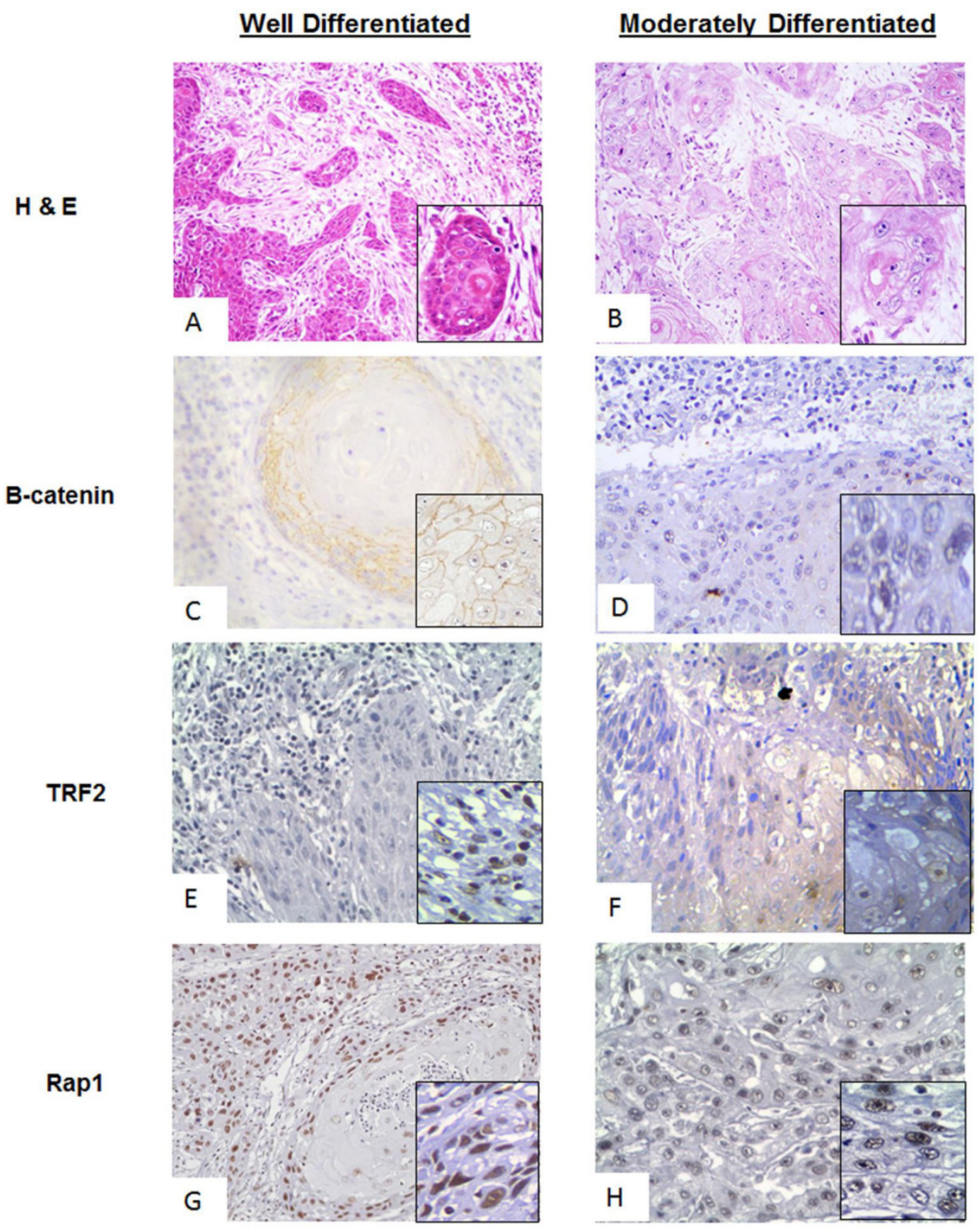

Figure 2: A comparative analysis of $\beta$-catenin, TRF2, Rapl expression in WDSCC and MDSCC by Immunohistochemistry. (A) Image representing the Haematoxylin and Eosin (H\& E) stain in well differentiated region. (B) Image representing the Haematoxylin and Eosin (H\& E) stain in moderately differentiated regions. (C) Image representing stable membranous expression of $\beta$-catenin in WDSCC. (D) Image representing a nuclear translocation (inset) of $\beta$-catenin in MDSCC. (E) Image representing stable TRF2 expression in WDSCC. (F) Image representing a cytoplasmic translocation of TRF2 in MDSCC regions. (G) Image showing stable expression of Rapl in the nucleus in WDSCC. $(H)$ Image showing equally stable expression of Rapl in the nucleus in MDSCC.

Figure 3: Significant signatures of genomic instability in higher grades of tumor

Anaphase bridge analysis which is a hall mark of genomic instability was performed for both tumor and cut margin Sections. It was observed that there was a net increase in the frequency of anaphase bridges when compared to the tumour cut margin areas generated with increasing grades of tumor as compared with the normal cut margin sections. MDSCC and poorly differentiated cells showed higher anaphase bridge percentage. (Figure 3A, 3B)

\section{Figure 4: Accelerated telomere shortening in the higher grades of HNSCC samples}

Paired adjacent tissues were observed to have longer telomeres than in the HNSCC tumor tissue. To gain further insights into the telomere length changes, we have used in situ analysis of telomere length by quantitative-fluorescence in situ hybridization on paraffin tissue. Quantitative-fluorescence in situ hybridization analysis yielded similar reductions in telomere lengths in tumor tissues compared with the adjacent tissues. The reduction of telomere length in early or premalignant stages is also associated with 
genome instability. MDSCC/Poorly differentiated cells showed shortening of telomeres. (Figure 4A, 4B).

\section{Figure 5: Quantitative expression of $\beta$-catenin in different grades of HNSCC cases}

To assess the clinical implication of $\beta$-catenin mRNA expression in HNSCC cases, the expression of this gene was analysed by real time quantitative PCR. A significant correlationship of the expression status of $\beta$-catenin in the cut margin and the tumor sample with their respective clinical prognosis was observed as shown in Figure 5. Almost all the samples had background expression of $\beta$-catenin in the cut margin regions and P31, P25, P29, P62, P28, P56 and P57 showed an upregulation of the $\beta$-catenin gene in the cut margins compared to its tumor tissues which also correlates with a bad prognosis of the respective patients. This confirms the infilteration of the $\beta$-catenin positive aggressive cells in the cut margin area.

A)
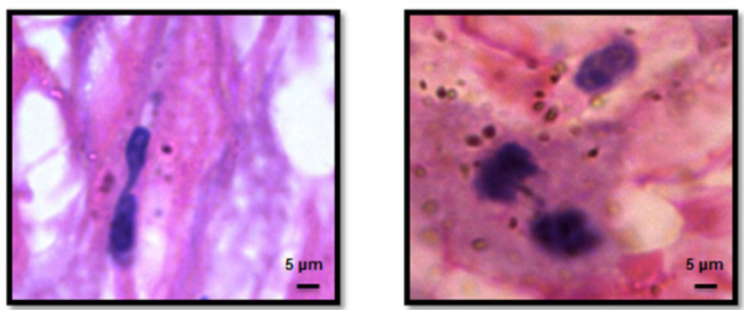

B)

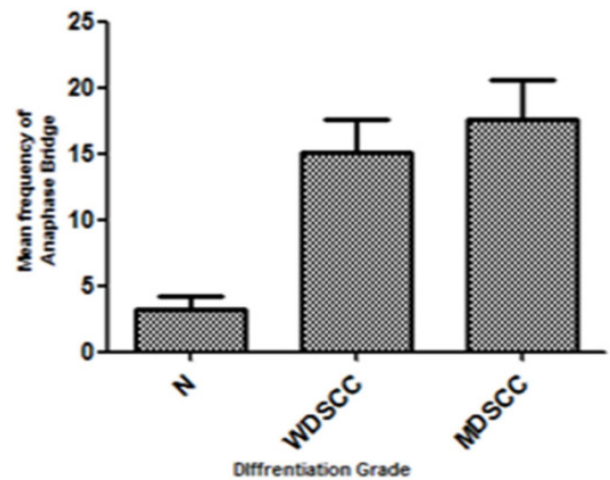

Figure 3: Incidence of anaphase bridges as an indicator of telomere dysfunction was found to be higher in higher grades of HNSCC. (A) Average frequency of anaphase bridge formation in all the grades of tumors. $* * * P<0.0001$. (B) Anaphase bridges in Haematoxylin and eosin (H\&E) stained tumor paraffin sections.

A)

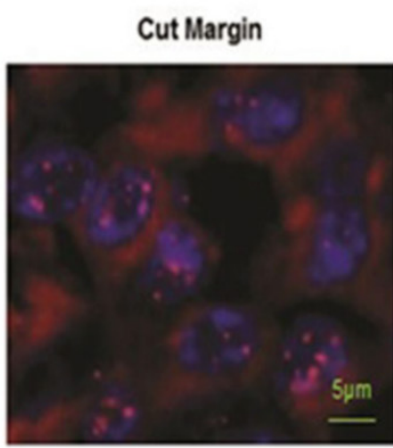

WDSCC

MDSCC

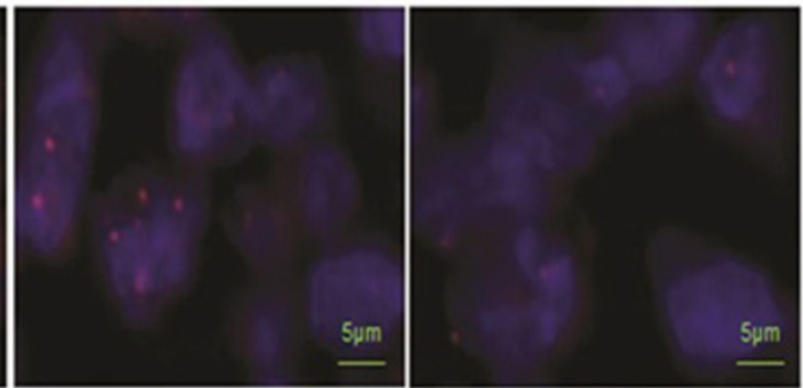

B)

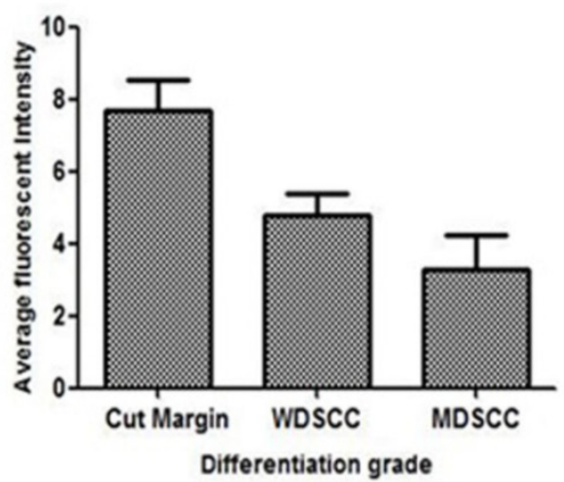

Figure 4: PNA-FISH revealed reduced telomere length in higher grades of tumor tissue sample. (A) Telomere length measured on paraffin tissue sections by PNA $\mathrm{FISH}$. Normal sections showed 7.7 as an average fluorescent intensity, WDSCC sections showed 4.8 fluorescent intensity, and MDSCC sections showed 3.3 fluorescent intensity as telomere length on an average when compared with the paired adjacent tissues. Bars indicate SE. (B) Representative pictures of tissue FISH in the paraffin sections of Normal, WDSCC and MDSCC tumors showing telomere signals using the telomere specific Cy-3 labelled PNA probe. $*$ P $<0.05$. The fluorescent intensity was calculated as arbitrary fluorescent intensity by Zeiss ISIS software. 


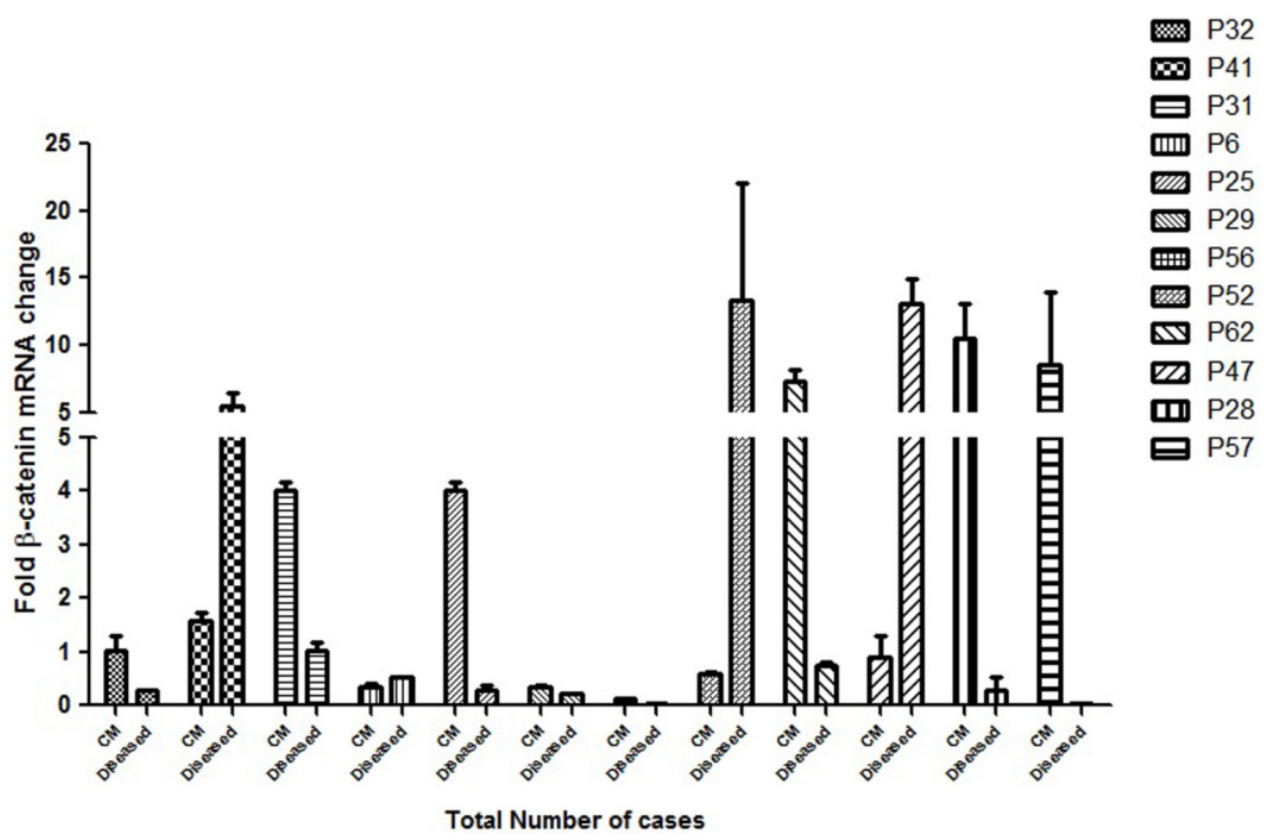

Figure 5: $q P C R$ analysis of the $\beta$-catenin gene expression as a factor of fold change in mRNA for cut margin (CM) and diseased tissues of 12 randomly chosen HNSCC cases are shown. P32 belonged to lower grade leukoplakia against which the expressions of the rest of the cases were compared. Upregulation of $\beta$-catenin in the cut margin region was observed in cases P31, P25, P29, P56, P62, P28 and P57 as compared to their respective counterparts which is suggestive of diseased cells infiltration expressing $\beta$-catenin into the cut margin area.

\section{Discussion}

Mutations in the wnt signalling pathway leads to alterations in the $\beta$-catenin level in the cells. ${ }^{[28]} \mathrm{Al}-$ tered/reduced $\beta$-catenin expression has been correlated with the higher histological grade, poor prognosis, aggressive phenotype, invasion and metastasis. ${ }^{[36]}$ Loss of $\beta$-catenin protein at the membrane thus leads to reduced intercellular adhesion associated with the loss of differentiation and acquisition of an invasive phenotype in a range of tumor. ${ }^{[37,38]}$ Genomic Instability is known to play key role in therapy outcomes in solid tumors as it is crucial in the process of carcinogenesis. ${ }^{[35]}$ In this study an attempt has been made to find observational correlation if there is any in between $\beta$-catenin, hTert and telomere group of proteins with respect to the grade specific differentiation, along with the variation in genomic instability in a cohort of 67 patients selected from our library of large tissue repository on the basis of stable beta catenin expression both in the tumor and their cut margin counterpart. Cases with stable $\beta$-catenin were selected for its major role in tumorigenesis as can be seen from our IHC data Figure 1.

Previous studies have shown that upregulation of the $W n t / \beta$-catenin signalling leads to upregulation of TRF2 level, key player of the shelterin complex, resulting in enhanced telomere protection. ${ }^{[39]}$ Simultaneously, $\beta$-catenin, escapes degradation on the activation of the Wnt signal on the cells promoting the expression of hTert in embryonic stem (ES) cells and cancer cells. ${ }^{[13]}$ The wnt/ $\beta$-catenin thus couples two different telomeric maintenance pathways that are activation of hTert and maintaining the TRF2 level.[39] In our study we observed similar pattern of occurrence for the $\beta$-catenin, hTert, Rap1 and TRF2 group of proteins in human HNSCC cases supported by the protein expression in tumor and the cut margin areas. The western blot data clearly indicates stabilization of $\beta$-catenin resulting in stable expression of hTert and TRF2 which are known as its direct transcriptional targets (Figure 1A). The quantified western blot data confirms the observation and shows a similar trend, the cut margin showing stable $\beta$-catenin expression indicates infiltration (Figure 1B). To validate the findings, Immunohistochemistry analysis was performed to confirm the expression of $\beta$-catenin in the infiltrating regions of tumor sections. The results showed a distinct membranous along with nuclear expression of $\beta$-catenin in the invading front (Figure $1 \mathrm{C}$ (II and III)) of the tumor when seen with respect to the normal squamous cell regions (Figure 1C (I)) yet to be invaded.

The cases were then categorized according to their histological differentiation grading as WDSCC and MDSCC respectively. To correlate the protein levels of $\beta$-catenin along with the shelterin component and their co-expression in a grade specific differentiation phenotype, we performed a comparative immunohistochemistry analysis in different grades of tumor cases as shown in Figure 2. Haematoxylin and eosin stain of both well differentiation and moder- 
ately differentiation tissue regions are shown in Figure $2 \mathrm{~A}$ and $2 \mathrm{~B}$. For the dedifferentiation to occur, $\beta$-catenin, being a cadherin group of protein, plays a vital role in metastasis which shows translocation from membranous cadherin expression to nuclear transcriptional factor localisation. ${ }^{[28]}$ In the present study $\beta$-catenin is showing a stable membranous expression in WDSCC and a nuclear translocation in MDSCC as illustrated in Figure $2 \mathrm{C}$ and 2D(inset). TRF2, being the transcriptional target of $\beta$-catenin and a shelterin group of protein is also stable in WDSCC and a cytoplasmic translocation is observed in MDSCC regions as seen in Figure 2E and 2F (inset). Rap1, a telomeric protein, is strongly and stably localised in the nucleus both in WDSCC and MDSCC confirming a telomere crisis and need to stabilize the telomere Figure $2 \mathrm{G}$ and $2 \mathrm{H}$.

With increasing histological grade the extent of DNA Damage is increased with a compromised repair process as a consequence of which the shelterin complex protecting the telomere also disrupts, signalling ATM-dependent DNA Damage sensors leading to genomic instability, a hallmark of tumorigenesis.[39] There was a sharp increase in the genomic instability parameter with increasing histological grade with respect to the frequency of anaphase bridges found in the patient cohort under consideration as shown in Figure 3.

The average anaphase bridge was $<18 \%$ in MDSCC when compared to $<13 \%$ in WDSCC and $<3 \%$ in normal cut margin of samples. It correlated well with decrease in Telomere length in moderately differentiated samples <4(arbitrary fluorescent unit) when compared to WDSCC and normal cut margin $>7$ as can be seen in Figure 4. It has been reported that telomere shortening is associated with aggressiveness, higher histological grade and poor prognosis of several type of cancer. ${ }^{[41,42]}$ With increasing histological grades, DNA damage is expected to be higher coupled with the loss of telomere length as compared to the lower grades thus indicating genomic instability and dedifferentiation. This accelerated telomere shortening is a hall mark of telomere deregulation and Sheltrin disintegration. ${ }^{[35]}$ Therefore evidence from our study supports the earlier known fact that telomere dysfunction impairs genetic stability and it correlates with the aggressiveness of the tumor.

To observe the correlationship of the $\beta$-catenin gene expression, 12 cases of different HNSCC grades were randomly selected from the cohort for the real time quantitative PCR analysis Figure 5. A stable $\beta$-catenin expression was observed both in the cut margin and their respective tumor tissues in the samples under consideration. There was a net upregulation of the $\beta$-catenin expression level in the majority of cut margins samples of the patients and more than $50 \%$ of the cases showed considerably high expression in the cut margin areas when compared to the tumor. A significant correlationship was observed in the cases showing a net upregulation in the $\beta$-catenin expression level in the cut margin and their respective pathological and clinical prognosis as highlighted in Additional File 1. From the cohort it was also observed that 13 patients out of 67 showed recurrence within 18 months of the onset of the disease. (Additional File 1) Strikingly, all of the recurrence cases had overall stable expression of $\beta$-catenin and telomere dysregulation, associated with poor prognosis and finally died. Although less in number but this confirms the infiltration of aggressive $\beta$-catenin upregulated cells in the cutmargin area in the patients having poorer pathological and clinical outcomes which results in minimal residual disease and recurrence.

\section{Conclusion}

In conclusion, $\beta$-catenin being the cadherin group of protein and a potent transcription factor plays a vital role in cellular differentiation and metastasis. Our molecular pathological validation study with patient samples has shown that $\beta$-catenin might be playing a vital role in regulating two very important pathways that are telomere maintenance and hTERT regulatory pathway and telomere protection shelterin proteins, known to be the hallmarks of tumorigenesis and responsible for the DNA Damage responses generated due to the progression of the carcinogenesis. With dedifferentiation there is a DNA damage microenvironment with an impaired repair process leading to severe genomic instability. Although it needs validation in large cohort of samples we have attempted to link three distinct and important events in molecular carcinogenesis processes such as tumour development, dedifferentiation and genomic instability in HNSCC tissue specimens graded by independent pathologists Figure 6 . As the process of tumorigenesis is understood at various levels and expression of molecular markers such as $\beta$-catenin, hTERT, TRF2, RAP1 correlates significantly with the process of tumorigenesis, we propose that molecular pathology along with classical WHO grading would be a better diagnostic and prognostic tool for effective management of HNSCC. 

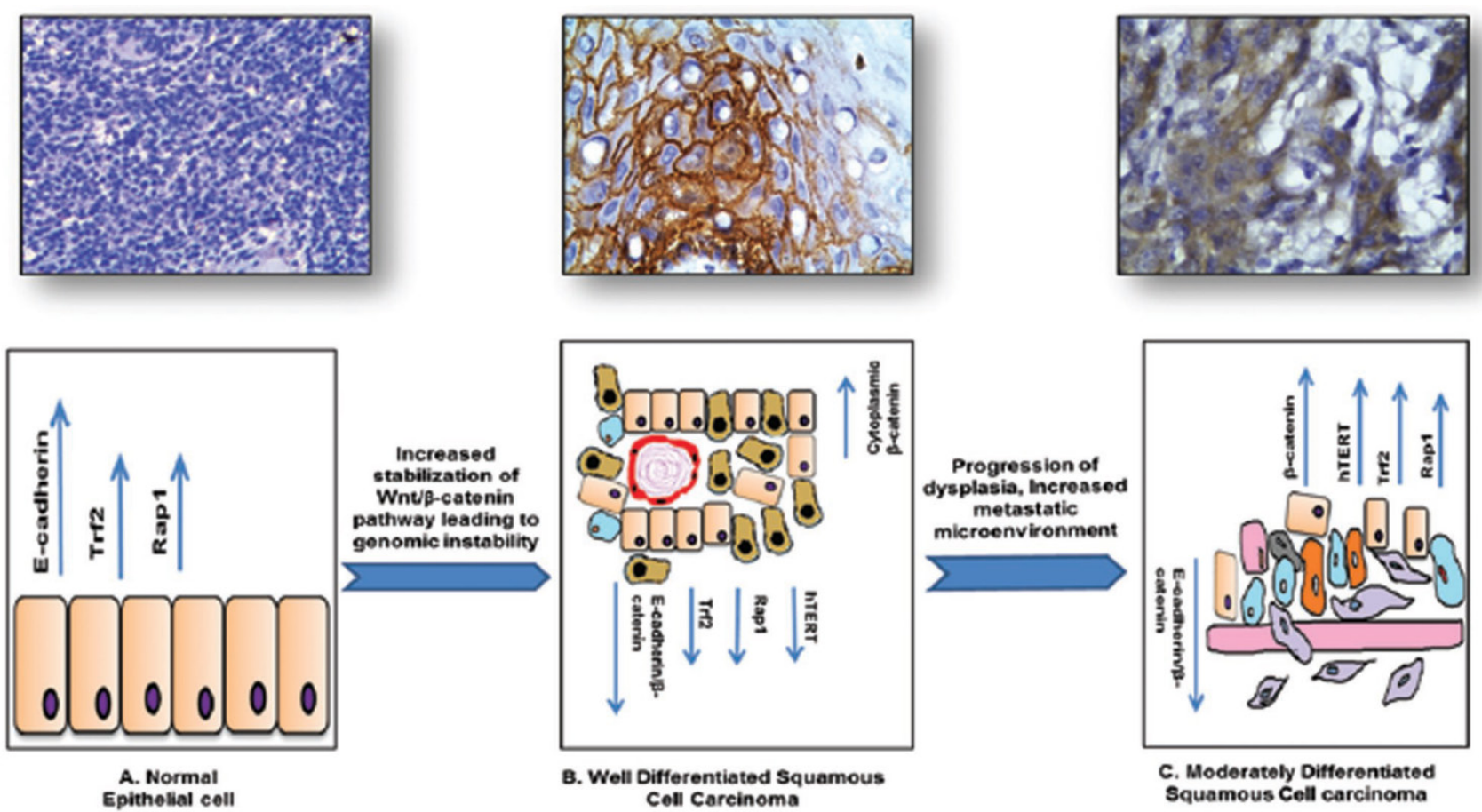

A. Normal Cell Carcinoma

C. Moderately Differentiated Squamous Cell carcinoma

C

Figure 6: Schematic representation of the regulatory proteins involved in the process of tumorigenesis and linking three important events such as tumor development, dedifferentiation and genome instability in HNSCC.

\section{Abbreviations}

HNSCC: Head and Neck Squamous Cell Carcinoma; MDSCC: Moderately Differentiated Squamous Cell Carcinoma; WDSCC: well differentiated squamous cell carcinoma; MRD: minimal residual disease; DSB: double stranded breaks.

\section{Supplementary Material}

\section{Additional File 1:}

Table showing clinico- pathological details of patients (staging, type of tumor, morbidity CT, RT planning and prognosis).

http://www.jcancer.org/v06p0192s1.xlsx

Additional File 2:

Figure S1: Western blot showing protein expression in the cut margin and tumor samples of the respective four antibodies ( $\beta$-catenin, TRF2, and hTERT) for 33 cases and $\beta$-catenin protein expression in the cut margin and tumor samples for additional 34 cases. Figure S2: Immunohistochemistry images showing protein expression cut margin and tumor samples of the respective antibodies ( $\beta$-catenin, TRF2) for 33 cases.

http://www.jcancer.org/v06p0192s2.pdf

\section{Acknowledgement}

This work was supported by extramural funding from Department of Biotechnology (DBT), Government of India.

\section{Conflict of Interest}

The authors declare no conflict of interest.

\section{References}

1. Song J, Chang I, Chen Z, Kang M, Wang C-Y. Characterization of Side Populations in HNSCC: Highly Invasive, Chemoresistant and Abnormal Wnt Signaling. PLoS ONE.2010; 5(7): e11456.

2. Saranath D. Cancer: a global view. Contemporary Issues in Oral Cancer. Oxford University Press. 2000: 1-29.

3. Pathare SM, Gerstung M, Beerenwinkel N, Schäffer AS, Kannan S et al.. Clinicopathological and prognostic implications of genetic alterations in oral cancers. Oncology Letters. 2011; 2: 445-451.

4. Nair U, Bartsch H and Nair J. Alert for an epidemic of oral cancer due to use of the betel quid substitute's gutkha and pan masala: a review of agents and causative mechanisms. Mutagenesis. 2004; 19: 251-262.

5. Rehman AO, Wang CY. CXC12/SDF-1a activates NF-kB and promotes oral cancer invasion through the Carma 3/Bcl10/Malt1 complex. Int J Oral Sci. 2009; 1: 105-118.

6. Rubinfeld B, Robbins P, El-Gamil M, Albert I, Porfiri E and Polakis P. Stabilization of beta-catenin by genetic defects in melanoma cell lines. Science. 1997; 275: $1790-1792$.

7. Reya T, Clevers H. Wnt signalling in stem cell and cancer. Nature.2005; 434(7035):843-50.

8. Iwai S, Yonekawa A, Harada C, Hamada M, Katagiri W, Nakazawa M and Yura Y. Involvement of the Wnt-B-catenin pathway in invasion and migration of oral squamous carcinoma cells. International Journal of Oncology.2010; 37: 1095-1103.

9. Burkert J, Wright NA, Alison MR. Stem cells and cancer: an intimate relationship. J Pathol. 2006; 209: 287-97. 
10. Yao H, Ashihara E, Maekawa T. Targeting the Wnt/b-catenin signaling pathway in human cancers. Expert Opin. Ther. Targets. 2011; 15(7): 1472-8222.

11. Kitagawa S, Hatakeyama S, Shirane M, Matsumoto M, Ishida N,Hattori K, Nakamichi I, Kikuchi A, Nakayama KI and Nakayama K. An F-box protein, FWD1, mediates ubiquitin dependent proteolysis of B-catenin. EMBO J.1999; 18: 2401-2410.

12. Morin PJ, Sparks AB, Korinck V, Barker N, Clevers H, Vogelstein B and Kinzler KW: Activation of beta-catenin-Tcf signaling in colon cancer by mutation in beta-catenin or APC. Science. 1997; 275: 1787-1790.

13. Hoffmeyer $\mathrm{K}$ et al. Wnt/b-Catenin Signaling Regulates Telomerase in Stem Cells and Cancer Cells. Science.2012; 336: 1549-1554.

14. Gardano L, Pucci F, Christian L, Bihan TL and Harrington L. Telomeres, a busy platform for cell signaling. Front Oncol. 2013; 3:146.

15. Sfeir,A.,and deLange,T. Removal of shelterin reveals the telomere end-protection problem. Science. 2012; 336: 593-597.

16. Greider, C. W., and Blackburn, E. H. A telomeric sequence in the RNA of Tetrahymena telomerase required for telomere repeat synthesis. Nature. 1989; 337: 331-337.

17. Kim NW, Piatyszek MA, Prowse KR, Harley CB, West MD, Ho PL, Coviello GM, Wright WE, Weinrich SL, Shay JW. Specific association of human telomerase activity with immortal cells and cancer. Science.1994;266: 2011-2015.

18. Cesare, A. J., and Reddel, R. R. Alternative lengthening of telomeres. Models, mechanisms, and implications. Nat. Rev. Genet. 2010; 11: 319-330.

19. Kronenwett U, Ploner A, Zetterberg A, Bergh J, Hall P, Auer G, Pawitan Y. Genomic instability and prognosis in breast carcinomas. Cancer Epidemiol Biomarkers Prev. 2006; 15(9):1630-5

20. Jallepalli PV, Lengauer C. Chromosome segregation and cancer: cutting through the mystery. Nat Rev Cancer. 2001; 1(2):109-17.

21. Grunberg SM et al. Treatment of poor-prognosis extensive disease small-cell lung cancer with an all-oral regimen of etoposide and cyclophosphamide - a Southwest Oncology Group clinical and pharmacokinetic study. Cancer Chemother Pharmacol.1999; 44(6):461-8.

22. M Jaskelioff et al. Telomerase deficiency and telomere dysfunction inhibit mammary tumor induced by polyomavirus middle $\mathrm{T}$ oncogene. Oncogene 2009; 28: 4225-4236.

23. Hanahan D, Weinberg RA. Hallmarks of cancer: the next generation. Cell.2011; 144(5):646-74.

24. Molinolo AA, Amornphimoltham P, Squarize $\mathrm{CH}$, Castilho RM, Patel V, Gutkind JS. Dysregulated molecular networks in head and neck carcinogenesis. Oral Oncol. 2009;45(4-5):324-34.

25. Murnane JP, Sabatier L. Chromosome rearrangements resulting from telomere dysfunction and their role in cancer. Bioessays. $2004 ; 26(11): 1164-74$.

26. Zhang Y, Toh L, Lau P, and Wang X. Human Telomerase Reverse Transcriptase (hTERT) Is a Novel Target of the Wnt/_-Catenin Pathway in Human Cancer. The Journal of Biological Chemistry. 2012; 287(39): 32494-32511.

27. Blackburn, E. H. Telomeres. Trends Biochem. Sci. 1991; 16: 378-381.

28. Laxmidevi LB, Angadi PV, Pillai RK and Chandreshekar C. Aberrant $\beta$-catenin expression in the histologic differentiation of oral squamous cell carcinoma and verrucous carcinoma: an immunohistochemical study. Journal of Oral Science. 2010; 52(4): 633-640.

29. Park, J. I et al. Telomerase modulates Wnt signalling by association with target gene chromatin. Nature. 2009; 460: 66-72.

30. Prince ME, Sivanandan R, Kaczorowski A, Wolf GT, Kaplan MJ. Identification of a subpopulation of cells with cancer stem cell properties in head and neck squamous cell carcinoma. Proc Natl Acad Sci U S A. 2007; 104: 973-978.

31. Liu C, Li Y, Semenov M, Han C, Baeg GH, Tan Y, Zhang Z, Lin X and He X: Control of $B$-catenin phosphorylation/degradation by a dual-kinase mechanism. Cell. 2002; 108: 837-847.

32. Olinolo AA, Amornphimoltham P, Squarize $\mathrm{CH}$, Castilho RM, Patel V, Gutkind JS. Dysregulated Molecular Networks in Head and Neck Carcinogenesis. Oral Oncol. 2009; 45: 324-334.

33. Alapati $\mathrm{D}$, Rong $\mathrm{M}$, Chen $\mathrm{S}$, Lin $\mathrm{C}, \mathrm{Li} \mathrm{Y}$ and $\mathrm{Wu} \mathrm{S}$. Inhibition of lrP5/6-mediated Wnt/ $\beta$-catenin signaling by Mesd attenuates hyperoxia-induced pulmonary hypertension in neonatal rats. Pediatric Research. 2013; 73(6): 719-725.

34. Chen S, Rong M, Platteau A, et al. CTGF disrupts alveolarization and induces pulmonary hypertension in neonatal mice: implication in the pathogenesis of severe bronchopulmonary dysplasia. Am J Physiol Lung Cell Mol Physiol. 2011; 300: L330-40.

35. Poonepalli A et al. Telomere-Mediated Genomic Instability and the Clinico-Pathological Parameters in Breast Cancer. Genes, Chromosomes and Cancer.2008; 47:1098-1109.

36. Tuominen V.J., et al. ImmunoRatio: a publicly available web application for quantitative image analysis of estrogen receptor (ER), progesterone receptor (PR), and Ki-67. Breast Cancer Research. 2010. 12:R56.

37. Yu Z, Weinberger PM, Provost E, Haffty BG, Sasaki C, Joe J, Camp RL, Rimm DL, Psyrri A. $\beta$-Catenin functions mainly as an adhesion molecule in patients with squamous cell cancer of the head and neck. Clin Cancer Res. 2005; 11: 2471-2477.

38. Jung CK et al. Diagnostic use of nuclear b-catenin expression for the assessment of endometrial stromal tumors. Modern Pathology.2008; 21:756-763.

39. Diala et al. Telomere protection and TRF2 expression are enhanced by the canonical Wnt signalling pathway. EMBO reports. 2013; 16: 1-8.
40. N.Deirdre et al. Quantitative single cell analysis of cell population dynamics during submandibular salivary gland development and differentiation. Biology Open. 2013; 2: 439-447.

41. McClintock B. The Stability of Broken Ends of Chromosomes in Zea Mays. Genetics. 1941; 26(2):234-82.

42. Hande MP et al. Telomere length dynamics and chromosomal instability in cells derived from telomerase null mice. J Cell Bio.1999; 144:589-601.

43. Szafarowski T, Szczepanski MJ. Cancer stem cells in head and neck squamouscell carcinoma. Otolaryngol Pol. 2014; 68(3):105-11. 